\title{
The Physician Clinical Support System-Buprenorphine (PCSS-B): a Novel Project to Expand/Improve Buprenorphine Treatment
}

\author{
James E. Egan, $\mathrm{MPH}^{7}$, Paul Casadonte, $\mathrm{MD}^{2}$, Tracy Gartenmann ${ }^{3}$, Judith Martin, MD ${ }^{4}$, \\ Elinore F. McCance-Katz, MD, PhD ${ }^{5}$, Julie Netherland, MSW' , John A. Renner, MD ${ }^{6,7}$, \\ Linda Weiss, $P h D^{7}$, Andrew J. Saxon, $M D^{8}$, and David A. Fiellin, $M D^{9}$
}

\begin{abstract}
${ }^{1}$ New York Academy of Medicine, New York, NY, USA; ${ }^{2}$ New York University Medical School, New York, NY, USA; ${ }^{3}$ American Society of Addiction Medicine (ASAM), Chevy Chase, MD, USA; ${ }^{4}$ BAART Turk Street Clinic, San Francisco, CA, USA; ${ }^{5}$ University of California, San Francisco, CA, USA; ${ }^{6}$ Boston University School of Medicine, Boston, MA, USA; ${ }^{7}$ VA Boston Healthcare System, Boston, MA, USA; ${ }^{8}$ Addictions Care Line VA Puget Sound Health Care System and The Department of Psychiatry and Behavioral Sciences University of Washington, Seattle, WA, USA;

${ }^{9}$ Yale University School of Medicine, New Haven, CT, USA.
\end{abstract}

Opioid dependence is largely an undertreated medical condition in the United States. The introduction of buprenorphine has created the potential to expand access to and use of opioid agonist treatment in generalist settings. Physicians, however, often have limited training and experience providing this type of care. Some physicians believe having a mentoring relationship with an experienced provider during their initial introduction to the use of buprenorphine would ease implementation. Our goal was to describe the development, implementation, resources, and evaluation of the Physician Clinical Support System-Buprenorphine (PCSS-B), a federally funded program to improve access to and quality of treatment with buprenorphine. We provide a description of the PCSS-B, a national network of 88 trained physician mentors with expertise in buprenorphine treatment and skills in clinical education. We provide information regarding the use the PCSS-B core services including telephone, email and in-person support, a website, clinical guidances, a warmline and outreach to primary care and specialty organizations. Between July 2005 and July 2009, 67 mentors and 4 clinical experts reported providing mentoring services to 632 participants in 48 states, Washington DC and Puerto Rico. A total of 1,455 contacts were provided through email (45\%), telephone (34\%) and in-person visits (20\%). Seventy-six percent of contacts addressed a clinical issue. Eighteen percent of contacts addressed a logistical issue. The number of contacts per participant ranged from 1-125. Between August 2005 and April 2009 there were 72,822 visits to the PCSS-B website with 179,678 pages viewed. Seven guidances were downloaded more than 1000 times. The warmline averaged more than 100 calls per month. The PCSS-B model provides support for a mentorship program to assist non-specialty physicians in the provision of buprenorphine and may serve as a model for dissemination of other types of care.

Received August 25, 2009

Revised December 3, 2009

Accepted April 14, 2010

Published online May 11, 2010
KEY WORDS: buprenorphine; opioid-related disorders; primary health care; education, distance.

J Gen Intern Med 25(9):936-41

DOI: $10.1007 / \mathrm{s} 11606-010-1377-y$

(C) The Author(s) 2010. This article is published with open access at Springerlink.com

$T$ he abuse of opioids, including heroin and prescribed pain medications, is a significant health problem in the United States. $^{1-3}$ Opioid agonist treatment decreases illicit opioid use, ${ }^{4-6}$ yet it is estimated that only $15 \%$ of those who need treatment receive it. ${ }^{7}$ The introduction of buprenorphine has created the potential to expand access to opioid agonist treatment to settings such as general psychiatry and primary care. $^{8}$

Buprenorphine, an opioid partial agonist, reduces opioid cravings, blocks the effects of opioids, and has limited overdose potential. Despite evidence of its efficacy and safety, ${ }^{9-13}$ officebased treatment using buprenorphine has not been as quickly adopted by U.S. physicians as some had anticipated or was needed. ${ }^{14-18}$ According to its manufacturer, approximately 585,000 individual patients (total) received medically supervised buprenorphine detoxification or maintenance treatment over the four year period from 2003 to $2007 .{ }^{19}$ During the same period of time approximately 250,000 patients per year received treatment with methadone. ${ }^{20}$ Buprenorphine treatment is facilitated through a relatively low number $(8,000$ to 10,000) of prescribers; therefore it represented a minority of the opioid agonist treatment provided during this period. ${ }^{21}$ In addition, almost half of prescribers are addiction specialists, ${ }^{21}$ suggesting limited uptake in more general medical practices such as general psychiatry or primary care.

Research on the uptake of buprenorphine suggests several factors influence physician interest in prescribing. These include knowledge and experience; availability of resources and support; cost and reimbursement; and perceptions regarding the challenges of caring for opioid-dependent patients. ${ }^{18,22-31}$

Most physicians who qualify to prescribe buprenorphine under the provisions of Drug Addiction Treatment Act (DATA 2000) do so by completing an 8-hour course on the treatment of opioid dependence. To date, more than 16,000 U.S. physi- 
cians have completed such a course. Recognizing that it is impossible to cover all the needed information in such a brief course, particularly for physicians with little pre-existing training in the clinical care of addictive disorders, the Substance Abuse and Mental Health Services Administration/ Center for Substance Abuse Treatment (SAMHSA/CSAT) funded the Physician Clinical Support System-Buprenorphine (PCSS-B) to assist with the implementation of buprenorphine in the U.S. treatment system. The goal of the PCSS-B is to promote more widespread use of buprenorphine by providing educational support to physicians interested in offering it as a treatment option for patients addicted to prescription opioids and heroin. The primary target audience was all physicians who had received initial 8-hour training but would benefit from additional educational support. By creating the educational support services of the PCSS-B, it was hoped that non-specialist physicians would be more inclined to undergo training.

The purpose of this article is to describe the development, implementation, resources, and evaluation of the PCSS-B for the first 4 years (July 2005-July 2009) of its implementation. Lessons from the remote mentoring model used within PCSS-B may be translatable to other medical and treatment issues.

\section{PCSS-B PROGRAM DEVELOPMENT AND DESCRIPTION}

Program Development. The development of the PCSS-B occurred according to established criteria in medical education and curriculum development. ${ }^{32}$ Needs analyses came from surveys conducted during early physician trainings ${ }^{28,31,33,34}$ and evaluations of clinical experience using buprenorphine. ${ }^{18}$ These surveys, conducted nationally on over 2,000 physicians, demonstrated that a substantial proportion of physicians receiving training were non-addiction specialists, had limited experience providing opioid agonist treatment, anticipated significant challenges and specific concerns with this model of care and were receptive to a mentorship model and support services that included online and distance learning techniques. This work, along with information regarding the characteristics of the learners derived from federal agencies and specialty medical societies, provided the rationale for the development of a training and support system comprised of multiple medical specialties (e.g. internal medicine, family practice, psychiatry, HIV medicine), treatment environments (e.g. inpatient, primary care, medication-free), practice locations (e.g. rural, urban) and practice types (e.g. private practice, academic, solo-practitioner).

Program Description. We identified specific instructional strategies based on observed deficits in knowledge, skills, and attitudes among the potential learners. The components of the PCSS-B are 1) a national network of trained physician mentors with expertise in buprenorphine treatment and skills in clinical education who are supported by a Medical Director and a small group of national Clinical Experts, 2) telephone warmline, 3) clinical guidances, 4) PCSS website (www. pcssmentor.org), and 5) targeted outreach to state chapters of primary care organizations. The efforts of the PCSS-B are overseen by a Steering Committee composed of representatives from leading addiction medicine societies, organizations that represent the target physician populations, and the federal government (Table 1).

As of late 2009, the PCSS-B included 88 mentors around the U.S. and Puerto Rico. The mentors were chosen by the Medical Director and the Clinical Experts based on the individual's knowledge of opioid dependence, experience using buprenorphine according to its FDA approved indications, teaching ability, and availability for contact with PCSS-B participants (those in need of mentor services) by telephone, email, and/or in person (to allow participants to observe their practice using buprenorphine).

The PCSS-B warmline is a national system of telephone triage that strives to answer queries about buprenorphine or match participants with mentors within 24 hours. Warmline staff, non-clinical administrative personnel with support from a nurse and physician, refer inquirers to the PCSS-B website and other technical assistance sources, assist in mentorparticipant matching according to specialty/geographic location/practice setting, respond to requests for general information and information about continuing medical education, and provide overall mentor-participant administrative support.

The PCSS-B has developed guidances to address common questions and concerns that have been posed by participants. The guidances (Table 2) are brief documents that provide concise and focused discussion of a clinical problem, briefly review the pertinent medical literature or consensus documents according to established levels of evidence, and provide clinical recommendations. All guidances are available through the PCSS-B website (www.pcssmentor.org). The website also provides resources on buprenorphine, including federal guidelines, links for additional resources and serves as a tool for data collection, data management, and project management (i.e. web-based surveys, electronic enrollment into the system and matching of mentors to participants).

The PCSS-B conducts outreach services to the organizations of steering committee members, state medical societies and state chapters of national organizations representing Internal Medicine and Family Physicians. These services include postings of descriptions of buprenorphine and PCSS$B$ services in electronic and paper media and providing speak-

Table 1. PCSS-B Steering Committee

\begin{tabular}{l}
\hline \hline Addiction Technology Transfer Centers \\
American Academy of Addiction Psychiatry \\
American Academy of Child and Adolescent Psychiatry \\
American Academy of Pediatrics \\
American Association for the Treatment of Opioid Dependence \\
American Medical Association \\
American Osteopathic Academy of Addiction Medicine \\
American Pain Society \\
American Psychiatric Association \\
American Society of Addiction Medicine \\
Association for Medical Education and Research in Substance Abuse \\
Center for Substance Abuse Treatment \\
Health Resources and Services Administration \\
National Alliance of Advocates for Buprenorphine Treatment \\
National Alliance of Methadone Advocates \\
National Association of State Alcohol/Drug Abuse Directors \\
National Institute on Drug Abuse/Clinical Trials Network \\
Pacific AIDS Education and Training Centers \\
Society of General Internal Medicine \\
The College on Problems of Drug Dependence \\
The New York Academy of Medicine
\end{tabular}

American Academy of Addiction Psychiatry

American Academy of Child and Adolescent Psychiatry

American Academy of Pediatrics

American Association for the Treatment of Opioid Dependence

American Pain Society

American Psychiatric Association

American Society of Addiction Medicine

National Alliance of Advocates for Buprenorphine Treatment

National Alliance of Methadone Advocates

National Association of State Alcohol/Drug Abuse Directors

Society of General Internal Medicine

The New York Academy of Medicine 


\section{Table 2. PCSS-B Clinical Guidances}

Adherence, Diversion and Misuse of Sublingual Buprenorphine Buprenorphine Induction

Management of Psychiatric Medications in Patients Receiving Buprenorphine/Naloxone

Monitoring of Liver Function Tests and Hepatitis in Patients Receiving Buprenorphine/Naloxone

Opioid Therapies, HIV disease and Drug Interactions

Physician Billing For Office-Based Treatment of Opioid Dependence

Pregnancy and Buprenorphine Treatment

Psychosocial Aspects of Treatment in Patients Receiving

Buprenorphine/Naloxone

Transfer from Methadone to Buprenorphine

Treatment of Acute Pain in Patients Receiving Buprenorphine/Naloxone

ers for presentations on opioid dependence and the use of buprenorphine at annual state chapter meetings. trends such as counts of unique visitors, pages viewed, hits, pages most often viewed, and documents downloaded.

All telephone calls to the warmline (but not calls to mentor physicians) and outreach to primary care activities are recorded and monitored by PCSS-B staff. Data are collected on a range of topics including mentor matching requests, numbers of requests per geographical region and inquiries related to the SAMHSA physician locator, a web-based tool that lists physicians who have completed the buprenorphine training and waiver process. Similarly, a PCSS liaison maintains a database of presentations conducted, PCSS resources distributed, and information published to promote PCSS-B services to primary care and the public.

Data are primarily collected using self-report through webbased surveys. Once submitted the data are transferred through a secure system to the evaluators at The New York Academy of Medicine. These data are then downloaded and imported into SPSS v.15 (SPSS Inc., Chicago IL) for analysis using descriptive statistics. Geographic data are analyzed using ArcGIS v.8.0 (ESRI, Redlands CA).

\section{EVALUATION METHODS}

Mentors are encouraged, but not required, to log all of their mentoring activities. As an incentive to provide accurate and timely data, mentors receive a modest honorarium (ranging from \$200-\$2,675/year) based on the number of contacts registered in a web-based system. Mentors can log into the web-based system and provide a description of contacts with their assigned participants. The system collects information on the geographic location of the contact, type of contact (urgent versus routine); nature of the questions (clinical or logistical), mode of contact (email, telephone or on-site visit), the clinical/ logistical areas addressed; and resources used.

Data on the use of PCSS-B clinical guidances are collected from mentors and using website statistics on downloaded documents. Website utilization data includes general usage

\section{RESULTS}

PCSS-B Mentors and Participants. The PCSS-B currently has 88 physician-mentors and five clinical experts located in 61 cities across 34 states and Puerto Rico. Nearly all mentors are certified in the treatment of addictions $(81 \%)$ and had more than three years of experience treating opioid dependence when they joined the PCSS-B (93\%). Mentor specialties include psychiatry (40\%), internal medicine (24\%), family medicine (15\%), and addiction medicine (14\%). Half identified their primary practice setting as an addiction treatment program, $25 \%$ a private practice and $22 \%$ an academic institution.

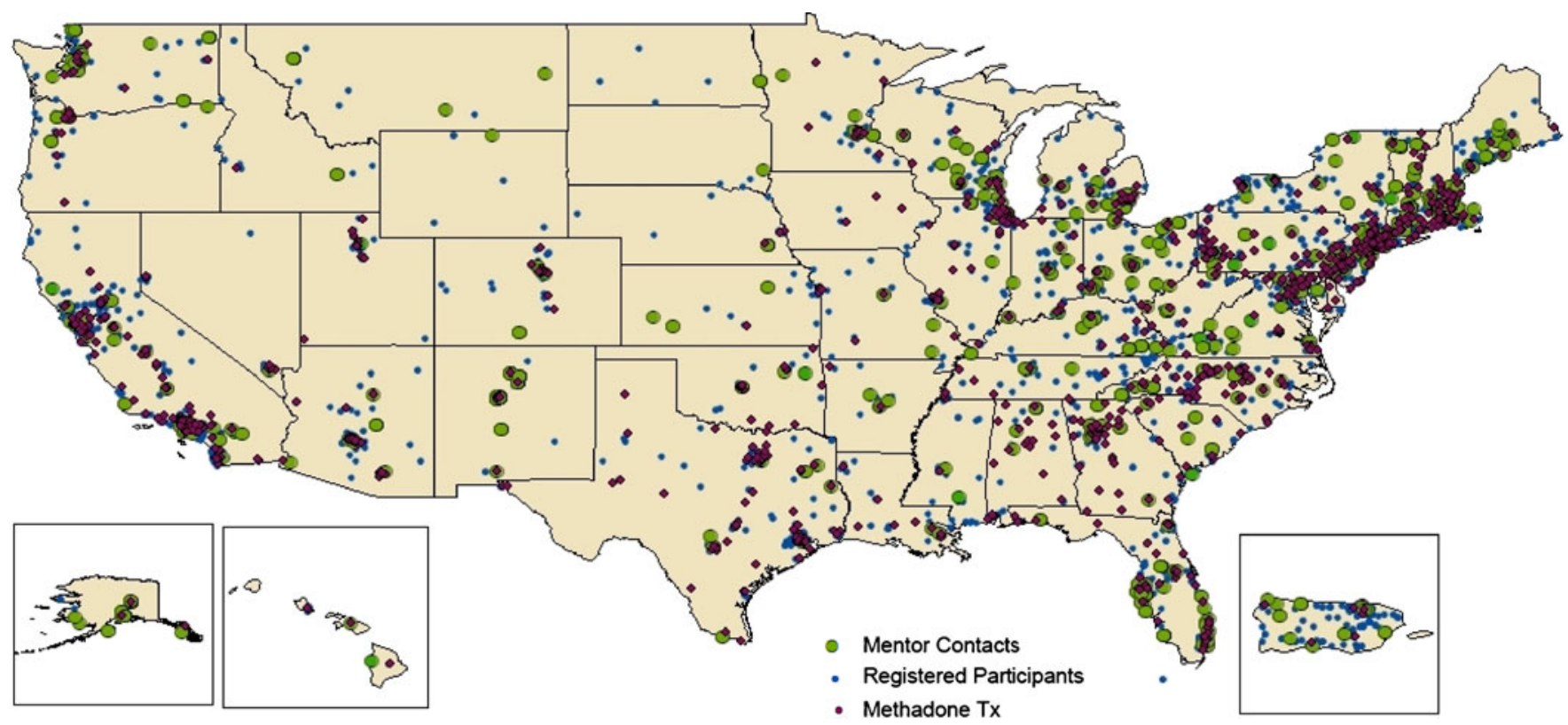

Figure 1. PCSS-B registered participants, contacts, and methadone treatment availability. 
As of July 2009, a total of 4,162 individuals have registered into the PCSS-B system representing all 50 states, Washington DC, and Puerto Rico (Fig. 1). PCSS-B participants identify their specialty as psychiatry (36\%), internal medicine (13\%), family medicine (22\%), addiction medicine $(3 \%)$, and other $(24 \%)$. Primary practice settings include private practice (49\%), academic setting $(8 \%)$, addiction treatment programs $(5 \%)$, and other (35\%). The majority of individuals (76\%) self-registered by phone or through the website or were automatically registered at a training designed to allow physicians to meet the requirements of DATA 2000. Far fewer (5\%) were registered into the system by a mentor. Mentor-registered participants, however, account for over half of the mentor contacts recorded.

PCSS-B Services Provided (mentor-participant contacts). Between July 2005 and July 2009, 67 mentors and four clinical experts reported providing mentoring services to 632 participants in 48 states (none in Iowa or Utah), Washington DC and Puerto Rico (Fig. 1). A total of 1,455 contacts were provided through email $(45 \%)$, telephone $(34 \%)$ and in-person visits $(20 \%)$. Mentors provided a wide range of services and support. Seventy six percent of contacts included a clinical issue and 18\% included a logistical issue. The most frequently requested clinical and logistical support is presented in Table 3 . The number of contacts per participant varied greatly, ranging from 1 to 125 . The majority of participants had five or fewer contacts. Mentors report spending an average of 5 minutes on preparation, 15 minutes of direct contact, and about 3 minutes on follow per contact.

Warmline. The PCSS-B warmline responds to an average of 100 inquiries and requests each month via email (55\% of inquires), phone (43\%), the website and fax (both < 1\%). Overall, just under half of the requests (49\%) were from individuals seeking a mentor and about a third (31\%) were requests for general information about the PCSS-B. Other requests included information about buprenorphine courses/trainings (7\%), continuing medical education (5\%), the buprenorphine physician locator (4\%), and how to become a mentor (3\%).

PCSS Clinical Guidances and Website Use. Mentors reported distributing PCSS-B clinical guidances in over $10 \%$ of contacts. Between August 2005 and April 2009 there were 72,822 visits to the PCSS-B website with 179,678 pages viewed. The pages most often viewed were those that included information on the PCSS-B, the mentors, or resources related to buprenorphine prescribing and seven guidances were downloaded more than 1,000 times (Table 4).

Outreach to Primary Care. From 2005-2008, mentors presented 57 educational workshops to state chapters of

Table 3. Most Frequently Provided Clinical and Logistical Support

\begin{tabular}{llll}
\hline Clinical & $\%^{*}$ & Logistical & $\%^{* *}$ \\
\hline Medication dose management & 34 & Scheduling & 43 \\
Dosing induction procedure & 32 & Payment & 35 \\
Induction procedure timing & 26 & Provider availability & 27 \\
Clinical logistical assistance & 19 & Paperwork & 24 \\
Chronic pain & 12 & & \\
\hline
\end{tabular}

$* \%$ of clinical contacts, $* * \%$ of logistical contacts
Table 4. Most Frequently Downloaded PCSS-B Website Documents

\begin{tabular}{|c|c|}
\hline Document & Downloaded \\
\hline $\begin{array}{l}\text { PCSS-B Guidance: Transfer from Methadone to } \\
\text { Buprenorphine }\end{array}$ & 2573 \\
\hline $\begin{array}{l}\text { PCSS-B Guidance: Treatment of Acute Pain } \\
\text { in Patients Receiving Buprenorphine/Naloxone }\end{array}$ & 1967 \\
\hline $\begin{array}{l}\text { PCSS-B Guidance: Pregnancy and Buprenorphine } \\
\text { Treatment }\end{array}$ & 1847 \\
\hline $\begin{array}{l}\text { PCSS-B Guidance: Monitoring of Liver Function } \\
\text { Tests and Hepatitis in Patients } \\
\text { Receiving Buprenorphine/Naloxone }\end{array}$ & 1735 \\
\hline $\begin{array}{l}\text { PCSS-B Guidance: Physician Billing For Office-Based } \\
\text { Treatment of Opioid Dependence }\end{array}$ & 1379 \\
\hline $\begin{array}{l}\text { PCSS-B Guidance: Management of Psychiatric } \\
\text { Medications in Patients Receiving } \\
\text { Buprenorphine/Naloxone }\end{array}$ & 1256 \\
\hline DSM IV: Instructions & 1062 \\
\hline $\begin{array}{l}\text { PCSS-B Guidance: Opioid Therapies, HIV Disease } \\
\text { and Drug Interactions }\end{array}$ & 1021 \\
\hline COWS - Clinical Opiate Withdrawal Scale & 930 \\
\hline $\begin{array}{l}\text { Tip 40: Clinical Guidelines for the Use of } \\
\text { Buprenorphine }\end{array}$ & 920 \\
\hline
\end{tabular}

American Academy of Family Physicians (AAFP), American College of Physicians (ACP), and state medical societies including a number of states in which access to opioid treatment is less readily available such as Alaska, Hawaii and North Dakota. Articles about the PCSS-B have appeared in over 35 different publications, including USA Today, American Medical News, American Academy of Family Practice News Now and Society of General Internal Medicine Forum, as well as 14 State Medical Association newsletters.

\section{Support for Opioid Treatment in Areas with Limited Availability} of These Services. Geographic data suggest that the PCSS-B has been successful at providing support for the treatment of opioid addiction to areas with little or no access to methadone. In some parts of the United States, buprenorphine offers the first and only access for pharmacologically-based treatment for opioid dependence. A goal of the PCSS-B was to promote the availability of buprenorphine across the country paying particular attention to those areas without reliable access to methadone treatment. The program has proven successful at providing support to physicians in several remote areas and in states that do not permit methadone maintenance treatment or have greatly limited access to methadone maintenance treatment for opioid dependence. This can be seen in the map (Fig. 1) which plots the locations of PCSS-B contacts and the availability of methadone treatment. ${ }^{35}$ In the 13 states with fewer than five locations to access methadone treatment (AK, AR, DE, HI, IA, ID, MS, MT, ND, NE, SD, TN, WY), mentors have had 100 contacts with PCSS-B participants. Additionally there is evidence to support the role of PCSS-B in providing support to providers in rural areas which lack methadone treatment opportunities.

\section{DISCUSSION}

Office-based buprenorphine treatment has the potential to expand access to and use of opioid agonist treatment in non- 
specialty settings. Recent research suggests several factors affect treatment uptake including: physician knowledge and experience; availability of resources and support; cost and reimbursement; and a perception of attracting patients with significant psychosocial needs. The PCSS-B was developed to address the first of these by improving physician knowledge and linking physicians to experienced prescribers. Our results indicate that the PCSS-B has been able to disseminate information regarding the appropriate use of buprenorphine through email, telephone, and in-person support to participants in 48 states, Washington DC, and Puerto Rico. In particular, support has focused on both clinical (medication dose management, dosing induction procedure, induction procedure timing, clinical logistical assistance, and chronic pain) and logistical (scheduling, payment, provider availability, paperwork, and medication supply) issues related to providing buprenorphine treatment.

Mentoring has been shown to be an effective method of transferring knowledge and skills in many contexts. While not addiction specific, the use of mentor relationships in the Association for Australian Rural Nurses' Mentor Development and Support Project resulted in enhanced knowledge, skills, and confidence among nurses in rural and remote areas. ${ }^{36}$ The shared care concept of United Kingdom is another approach that has shown some success in promoting addiction treatment in primary care. ${ }^{37}$ In one pilot program, specialists nurses trained in addiction were successfully used in rural primary care to provide support to general practitioners. In this instance nurses were able to provide important support and expertise to physicians who lacked addiction training, reduce physician workload, improve communication with pharmacists, and improve client satisfaction. ${ }^{38}$ The PCSS-B was inspired and components modeled after the National HIV/ AIDS Clinicians' Consultation Center established in the early 1990's to support HIV treatment. ${ }^{39}$

Our data are subject to several limitations. First, as a descriptive study, without a control condition or patient level data, we are not able to assess the true impact of the PCSS-B on care quality or buprenorphine treatment expansion. As such it is not possible to quantify the impact of the PCSS-B on population health. Nonetheless, anecdotal information derived from the mentors supports an interpretation that for those participants who accessed the system, buprenorphine treatment was facilitated and likely more consistent with guidelines. Second, while the sample incorporates a diverse range of physicians with regard to geography, practice setting, clinical background and experience, they are not representative of all physicians trained to prescribe buprenorphine. The findings are further limited by the fact that all data collected were by self-report using a web-based evaluation system. The burden of reporting services rested entirely on the physician mentors. While the web-based system was designed to be straight forward, the technology and time required to catalogue events proved challenging for some. We note that this program did not provide market-value reimbursement to mentors for their time and effort and believe that many mentors were motivated to participate based on their commitment to the field and concern over the appropriate implementation of buprenorphine treatment. Based on conversations with mentors, we estimate that we were able to collect $33-50 \%$ of the total PCSS-B mentor-participant contacts. To improve data collection, the mechanism of apportioning honorariums to mentors has been modified to more directly reflect the number of recorded contacts. It appears that not all registered participants went on to receive mentoring services and that participants who were registered by mentors had the highest use of services. This implies that strategies to foster a close connection between participants and mentors may increase use of PCSS-B services.

Despite these limitations, these data do provide important insight into physician educational needs with respect to officebased opioid treatment. They also demonstrate the feasibility of implementing a national system of mentors positioned to provide ongoing education and support to facilitate and promote addiction treatment in non-specialty treatment settings. We believe the most immediate lessons that have been learned from the PCSS-B are the feasibility, acceptability, and use of expert mentoring services via email, telephone, and on-site visitation in the practice of medicine. These types of support services may be most appropriate in situations in which knowledge, skills and attitudes are not learned in medical school or residency, yet are needed by practicing clinicians. The examples of HIV care and buprenorphine treatment are emblematic of this type of need. Based on the experience with the PCSS-B, SAMHSA/CSAT have funded the implementation of a parallel program designed to improve the quality of care among patients receiving methadone in opioid treatment programs, pain practices, and primary care-the Physician Clinical Support System for Methadone (PCSS-M). For more information on this program see www.pcssmethadone.org.

Acknowledgements: The Physician Clinical Support SystemBuprenorphine (PCSS-B) project is funded by the Substance Abuse and Mental Health Services Administration, Center for Substance Abuse Treatment. Dr. Fiellin is supported by the National Institute on Drug Abuse (NIDA RO1 DA019511, RO1 DA025991, RO1 DA020576). We thank Michael L. Green, M.D., M.Sc. for his thoughtful review of an earlier version of this manuscript.

Conflicts of Interest: Judith Martin is employed by BAART Programs, a substance abuse treatment program which provides treatment to patients with buprenorphine. Elinore McCance-Katz is on the Data Safety Monitoring Board of Synosia. John Renner has consulted as an author and Advisory Board member with Shire Pharmaceuticals; has received honoraria from Alkermes/Cephalon, Forest Labs, and Reed Medical Education; and owns Johnson \& Johnson stock. Andrew Saxon has consulted with Reckitt Benckiser Corporation; has received honoraria from Direct Success, Inc. and Schering-Plough Canada; has received a grant and has another pending from Titan Pharmaceuticals. David Fiellin serves on an expert advisory board to monitor for misuse, abuse, and diversion of buprenorphine for Pinney Associates.

Open Access: This article is distributed under the terms of the Creative Commons Attribution Noncommercial License which permits any noncommercial use, distribution, and reproduction in any medium, provided the original author(s) and source are credited.

Corresponding Author: James E. Egan, MPH; New York Academy of Medicine, 1216 5th Avenue, New York, NY 10029, USA (e-mail: jegan@nyam.org).

\section{REFERENCES}

1. Substance Abuse and Mental Health Services Administration. Overview of findings from the 2003 national survey on drug use and health. Rockville: Substance Abuse and Mental Health Services Administration; 2004. 
2. Substance Abuse and Mental Health Services Administration. National household survey on drug abuse. Substance Abuse and Mental Health Services Administration; 2006.

3. Substance Abuse and Mental Health Services Administration. Overview of findings from the 2007 national survey on drug use and health. Rockville: Substance Abuse and Mental Health Services Administration; 2008.

4. Fiellin DA, Kleber H, Trumble-Hejduk JG, McLellan TA, Kosten TR. Consensus statement on office-based treatment of opioid dependence using buprenorphine. J Subst Abuse Treat. 2004;27:153-9.

5. Laine C, Hauck Ww, Gourevitch MN, Cohen A, Turner BJ. Regular outpatient medical and drug abuse care and subsequent hospitalization of persons who use illicit drugs. JAMA. 2001;285(18):2355-62.

6. O'Connor PG, Fiellin DA. Pharmacologic treatment of heroin-dependent patients. Ann Intern Med. 2000;133(1):40-54.

7. Merrill JO, Rhodes LA, Deyo RA, Marlatt GA, Bradley KA. Mutual mistrust in the medical care of drug users: the keys to the "narc" cabinet. J Gen Intern Med. 2002;17(5):327-33.

8. Fiellin DA, O'Connor PG, Chawarski M, Schottenfeld RS. Processes of care during a randomized trial of office-based treatment of opioid dependence in primary care. Am J Addict. 2004;13(Suppl 1):67-78.

9. Fiellin DA, O'Connor P. New federal initiatives to enhance the medical treatment of opioid dependence. Ann Intern Med. 2002;137(8):688-92.

10. Gibson AE, Doran CM, Bell JR, Ryan A, Lintzeris N. A comparison of buprenorphine treatment in clinic and primary care settings: a randomised trial. Med J Aust. 2003;179(1):38-42.

11. Novick DM, Joseph H, Salsitz EA, et al. Outcomes of treatment of socially rehabilitated methadone maintenance patients in physicians' offices (medical maintenance): follow-up at three and a half to nine and a fourth years. J Gen Intern Med. 1994;9(3):127-30.

12. O'Connor PG, Samet JH. The substance-using human immunodeficiency virus patient: approaches to outpatient management. Am J Med. 1996;101(4):435-44.

13. O'Connor PG, Oliveto AH, Shi JM, et al. A randomized trial of buprenorphine maintenance for heroin dependence in a primary care clinic for substance users versus a methadone clinic. Am J Med. 1998;105(2):100-5.

14. National Consensus Development Panel on Effective Medical Treatment of Opiate Addiction: Effective medical treatment of opiate addiction. JAMA. 1998;280(1936-1943)

15. National Poll of Physicians Finds Barriers to Widespread Buprenorphine Use. Boston, MA: Join Together, Boston University School of Public Health; 2003.

16. Knudsen HK, Ducharme LJ, Roman PM. The adoption of medications in substance abuse treatment: associations with organizational characteristics and technology clusters. Drug Alcohol Depend. 2006;164-74.

17. Knudsen HK, Ducharme LJ, Roman PM. Early adoption of buprenorphine in substance abuse treatment centers: data from the private and public sectors. J Subst Abuse Treat. 2006;30(4):363-73.

18. Stanton A, McLeod C, Luckey B, Kissin W, Sonnefled L. Expanding treatment of opioid dependence: initial physician and patient experiences with the adoption of buprenorphine. 2006. Available at: www. buprenorphine.samhsa.gov/ASAM_06_Final_Results.pdf. Accessed: March 10, 2010

19. Renner J. Education status report: successes \& challenges. Personal communication 2-21-2008 (slide set).

20. Substance Abuse and Mental Health Services Administration Office of Applied Studies. National Survey of Substance Abuse Treatment Services (N-SSATS): 2007. Data on Substance Abuse Treatment Facilities,
DASIS Series: S-44. Rockville, MD; 2008. Report No.: DHHS Publication No. (SMA) 08-4348.

21. Fiellin DA. The first three years of buprenorphine in the united states: experience to date and future directions. Journal of Addiction Medicine. 2007;1(2):62-7.

22. Netherland J, Botsko M, Egan JE, et al. Factors affecting willingness to provide buprenorphine treatment. J Subst Abuse Treat. 2009;36(3):24451 .

23. Cunningham CO, Sohler NL, McCoy K, Kunins HV. Attending physicians' and residents' attitudes and beliefs about prescribing buprenorphine at an urban teaching hospital. Fam Med. 2006;38(5):336-40.

24. Elliott V. Links between primary care, addiction services may help treatment. American Medical News (amednews.com). 1-29-0001.

25. Fiellin DA, O'Connor PG, Chawarski M, Pakes JP, Pantalon MV Schottenfeld RS. Methadone maintenance in primary care: a randomized controlled trial. JAMA. 2001;286(14):1724-31.

26. Fiellin DA, Barthwell A. Guideline development for office-based pharmacotherapies for opioid dependence. J Addict Dis. 2003;22: 109-20.

27. Godden T, Byrne A, Wanigaratne S, Feinmann C. Care and shared care of opiate misusers by general practitioners in inner London. J Subst Misuse Nursing, Health Social Care. 1997;2(4):217-21.

28. Sullivan L, Tetrault J, Bangalore D, Fiellin DA. Training HIV physicians to prescribe buprenorphine for opioid dependence. Subst Abuse. 2006;27(3):11-6.

29. Sullivan LE, Fiellin DA. Buprenorphine: its role in preventing HIV transmission and improving the care of HIV-infected patients with opioid dependence. Clin Infect Dis. 2005;41(6):891-6.

30. Turner BJ, Laine C, Lin YT, Lynch K. Barriers and facilitators to primary care or human immunodeficiency virus clinics providing methadone or buprenorphine for the management of opioid dependence. Arch Intern Med. 2005;165(15):1769-76.

31. West JC, Kosten TR, Wilk J, et al. Challenges in increasing access to buprenorphine treatment for opiate addiction. Am J Addict. 2004;13 (Suppl 1):8-16

32. Green ML. Identifying, appraising, and implementing medical education curricula: a guide for medical educators. Ann Intern Med. 2001;135 (10):889-96.

33. Gunderson EW, Fiellin DA, Levin FR, Sullivan LE, Kleber HD. Evaluation of a combined online and in person training in the use of buprenorphine. Subst Abus. 2006;27(3):39-45.

34. Wong JG, Holmboe ES, Jara GB, Martin J, Becker WC, Fiellin DA. Faculty development in small-group teaching skills associated with a training course on office-based treatment of opioid dependence. Subst Abuse. 2004;25(4):35-40.

35. Substance Abuse and Mental Health Services Administration. Substance abuse treatment facility locator. Available at: http://findtreat ment.samhsa.gov. Accessed: March 10, 2010.

36. Mills J, Lennon D, Francis K. Contributing to a culture of learning: a mentor development and support project for Australian rural nurses. Int J Nurs Pract. 2007;13(6):393-6.

37. Hampson JP, Roberts RI, Morgan DA. Shared care: a review of the literature. Fam Pract. 1996;13(3):264-79.

38. Smith E, Mistral W. Shared care: lessons from one model of shared care nursing in primary care. Drugs: Education, Prevention \& Policy. 2003;10 (3):263-70.

39. Liljestrand P, Goldschmidt RH. National HIV telephone consultation line [comment]. J Gen Intern Med. 1998;2(13): 146. 